PROCEEDINGS OF THE

AMERICAN MATHEMATICAL SOCIETY

Volume 128 , Number 12, Pages $3601-3608$

S 0002-9939(00)05519-2

Article electronically published on June 7, 2000

\title{
PYRAMIDAL VECTORS AND SMOOTH FUNCTIONS ON BANACH SPACES
}

\author{
R. DEVILLE AND E. MATHERON
}

(Communicated by Dale Alspach)

\begin{abstract}
We prove that if $X, Y$ are Banach spaces such that $Y$ has nontrivial cotype and $X$ has trivial cotype, then smooth functions from $X$ into $Y$ have a kind of "harmonic" behaviour. More precisely, we show that if $\Omega$ is a bounded open subset of $X$ and $f: \bar{\Omega} \rightarrow Y$ is $C^{1}$-smooth with uniformly continuous Fréchet derivative, then $f(\partial \Omega)$ is dense in $f(\bar{\Omega})$. We also give a short proof of a recent result of P. Hájek.
\end{abstract}

This note is motivated by recent results of P. Hájek ([H1], [H2]) concerning (Fréchet) smooth nonlinear operators on the space $c_{0}$.

In [H1], Hájek proved (among other things) that if $f: c_{0} \rightarrow \mathbb{R}$ is a $C^{1}$-smooth map with uniformly continuous derivative on $B_{c_{0}}$, then $f^{\prime}\left(B_{c_{0}}\right)$ is a relatively compact subset of $l_{1}$. From this, he deduced that if $Y$ is a Banach space with non trivial type and $f: c_{0} \rightarrow Y$ is $C^{1}$-smooth with locally uniformly continuous derivative, then $f$ is locally compact, which means that each point $x \in c_{0}$ has a neighbourhood $V$ such that $f(V)$ is relatively compact in $Y$. In [H2], he also proved that the same is true if $Y$ has an unconditional basis and does not contain $c_{0}$. These striking results are to be compared with another recent theorem, due to S. M. Bates ([B] $)$, according to which for any separable Banach space $Y$ there exists a $C^{1}$-smooth surjection from $c_{0}$ onto $Y$; clearly, such a map cannot be locally compact unless $Y$ is finite-dimensional, by the Baire category theorem.

This note is a by-product of several vain attempts to generalize Hájek's local compactness results to all Banach spaces $Y$ not containing $c_{0}$.

We show that if $X, Y$ are Banach spaces such that $Y$ has finite cotype and $X$ does not have finite cotype, then smooth functions from $X$ into $Y$ have a kind of "harmonic" behaviour (Theorem 1). We also prove that if $Y$ has finite cotype, then smooth functions from $c_{0}$ into $Y$ essentially turn weakly convergent sequences into (norm) Cesaro-convergent sequences (Theorem 2). Both results rest on an elementary finite-dimensional lemma (Lemma 1) involving what we have called pyramidal vectors of $c_{0}$ (Definition 1 ). Finally, we give a very short proof of Hájek's basic result for scalar-valued functions, which looks rather different (at least in its form) from the original one. This proof is based on the notion of strong sequential continuity (Definition 3), which might be of independent interest.

Let us now fix the notation that will be used throughout this note. The letters $X, Y$ will always designate (real) Banach spaces. If $Z$ is a normed space, we denote

Received by the editors February 19, 1999.

2000 Mathematics Subject Classification. Primary 46B20.

(C)2000 American Mathematical Society 
by $B_{Z}$ the closed unit ball of $Z$. For any set $B \subseteq Z$, we denote by $C_{u}^{1}(B, Y)$ the set of all maps $f: Z \rightarrow Y$ which are $C^{1}$ - smooth on some neighbourhood of $B$ with uniformly continuous derivative on $B$. If $\omega$ is a modulus of continuity, we put $C^{1, \omega}(B, Y)=\left\{f \in C_{u}^{1}(B, Y) ; \forall u, v \in B\left\|f^{\prime}(u)-f^{\prime}(v)\right\| \leq \omega(\|u-v\|)\right\}$; and if $M>0$, we let $C^{1, \omega, M}(B, Y)=\left\{f \in C^{1, \omega}(B, Y) ;\left\|f^{\prime}(x)\right\| \leq M\right.$ on $\left.B\right\}$. Finally, we denote by $\left(e_{i}\right)_{i \geq 0}$ the canonical basis of $c_{0}$, and by $c_{00}$ the linear span of the $e_{i}$ 's; and if $N$ is a positive integer, we put $l_{\infty}^{N}=\operatorname{span}\left\{e_{i} ; 0 \leq i \leq N-1\right\}$.

Definition 1. Let $K$ be a positive integer. We say that $a \in c_{0}$ is a $K$-pyramidal vector if one can write $a=\sum_{i=1}^{r} \lambda_{i} \mathbf{1}_{A_{i}}$, where $r \leq K,\left(A_{1}, \ldots A_{r}\right)$ is a decreasing sequence of (nonempty) finite intervals of $\mathbb{N}$ such that $\min A_{i}<\min A_{i+1}$ if $i \leq r-1$, and $\left.\left.\lambda_{1}, \ldots, \lambda_{r} \in\right] 0 ; 1 / K\right]$.

Recall that a Banach space $Z$ is said to have cotype $q(2 \leq q \leq \infty)$ if there is a numerical constant $C$ such that $\|\mathbf{z}\|_{l_{q}(Z)} \leq C\left\|\sum \varepsilon_{i} z_{i}\right\|_{L_{q}(Z)}$ for all finite sequences $\mathbf{z}=\left(z_{1}, \ldots z_{n}\right) \subseteq Z$ (where $\left(\varepsilon_{i}\right)$ is the sequence of Rademacher functions).

Lemma 1. Assume that $Y$ has finite cotype $q$. Let $\omega$ be a modulus of continuity and let $M>0$. Given a positive integer $K$ and $\varepsilon>0$, there exists a positive integer $N$ satisfying the following property: for any open set $V \subseteq l_{\infty}^{N}$ such that $0 \in V \subseteq B_{l_{\infty}^{N}}$ and for any $f \in C^{1, \omega, M}(\bar{V}, Y)$, one can find a $K$-pyramidal vector a such that

$$
a \in \partial V \text { and }\|f(a)-f(0)\|<\varepsilon+\omega(1 / K) .
$$

Proof. We will use the hypothesis on $Y$ in the following way: for any bounded linear operator $T: c_{0} \rightarrow Y$ and any positive number $\alpha$, the number of integers $i$ such that $\left\|T e_{i}\right\| \geq \alpha$ does not exceed $C_{q}^{q} \frac{\|T\|^{q}}{\alpha^{q}}$, where $C_{q}$ is the cotype constant of $Y$. This is easy to check.

Fix a positive integer $K$ and $\varepsilon>0$.

We choose a sequence of positive integers $\left(N_{0}, \ldots, N_{K}\right)$ such that for $i \leq K-1$, $N_{i}$ is "much greater" than $N_{i+1}$. More precisely, the $N_{i}$ 's are selected in such a way that

$$
\frac{N_{0}-C N_{1}^{q} / \varepsilon^{q}}{1+C N_{1}^{q} / \varepsilon^{q}} \geq 1+N_{1}, \ldots, \frac{N_{K-1}-C N_{K}^{q} / \varepsilon^{q}}{1+C N_{K}^{q} / \varepsilon^{q}} \geq 1+N_{K}
$$

where $C=C_{q}^{q} M^{q}$. Finally, we put $N=N_{0}$.

Now, fix an open set $V \subseteq l_{\infty}^{N}$ such that $0 \in V \subseteq B_{l_{\infty}^{N}}$ and a function $f \in$ $C^{1, \omega, M}(\bar{V}, Y)$.

Let us say that a decreasing sequence $\left(A_{0}, \ldots A_{p}\right)(0 \leq p \leq K)$ of subintervals of $[0 ; N[$ is admissible if the following properties are satisfied:

(i) $A_{0}=\left[0 ; N\left[\right.\right.$ and each $A_{i}$ has cardinality $N_{i}$.

(ii) For all $i \geq 1\left[a_{i-1} ; a_{i}\right] \subseteq V$, where $a_{0}=0$ and $a_{l}=\frac{1}{K} \sum_{j=1}^{l} \mathbf{1}_{A_{j}}$ if $l \geq 1$.

(iii) If $i \geq 1$, then $\left\|f^{\prime}\left(a_{i-1}\right) \cdot e_{l}\right\|<\frac{\varepsilon}{N_{i}}$ for all $l \in A_{i}$.

Notice that $\left(A_{0}\right)=([0 ; N[)$ is admissible, and that there is no admissible sequence of length $K+1$, because $V \subseteq B_{l_{\infty}^{N}}$.

Let $\left(A_{0}, \ldots, A_{p}\right)$ be an admissible sequence of maximal length. Then $p \leq K-1$; hence, by the choice of the sequence $\left(N_{0}, \ldots, N_{K}\right)$, it is possible to find an interval 
$A_{p+1} \subseteq A_{p}$ of cardinality $N_{p+1}$ such that $\min A_{p+1}>\min A_{p}$ and

$$
\forall l \in A_{p+1} \quad\left\|f^{\prime}\left(a_{p}\right) \cdot e_{l}\right\|<\varepsilon / N_{p+1} .
$$

Since $\left(A_{0}, \ldots A_{p+1}\right)$ cannot be admissible, this implies that the segment $I=\left[a_{p} ; a_{p}+\right.$ $\left.\frac{1}{K} \mathbf{1}_{A_{p+1}}\right]$ is not contained in $V$; and since $a_{p} \in V, I$ must intersect $\partial V$.

Let $\lambda=\min \left\{t \geq 0 ; a_{p}+t \mathbf{1}_{A_{p+1}} \in \partial V\right\}$, and put $a=a_{p+1}=a_{p}+\lambda \mathbf{1}_{A_{p+1}}$.

Since $\lambda \in] 0 ; 1 / K], a$ is a $K$-pyramidal vector, and of course $a \in \partial V$.

Moreover, if we put $h_{i}=a_{i}-a_{i-1}(1 \leq i \leq p+1)$, then $\left\|f^{\prime}\left(a_{i-1}\right) . h_{i}\right\|<\varepsilon / K$ and $\left\|h_{i}\right\| \leq 1 / K$ for all $i$, whence

$$
\begin{aligned}
\left\|f\left(a_{i}\right)-f\left(a_{i-1}\right)\right\| & \leq\left\|f^{\prime}\left(a_{i-1}\right) \cdot h_{i}\right\|+\left\|h_{i}\right\| \omega\left(\left\|h_{i}\right\|\right) \\
& <\varepsilon / K+1 / K \omega(1 / K)(1 \leq i \leq p+1) .
\end{aligned}
$$

Therefore, we get

$$
\begin{aligned}
\|f(a)-f(0)\| & \leq \sum_{i=1}^{p+1}\left\|f\left(a_{i}\right)-f\left(a_{i-1}\right)\right\| \\
& <\varepsilon+\omega(1 / K) .
\end{aligned}
$$

Remark. Let us give a geometrical interpretation of the above proof. For simplicity, assume that $V=B_{l_{\infty}^{N}}$. The points $a_{i}$ satisfy

$$
\left\|a_{i+1}-a_{i}\right\|=\left\|h_{i+1}\right\|=1 / K \quad \text { and } \quad\left\|a_{i}\right\|=i / K,
$$

so they form a path joining 0 to the unit sphere of $c_{0}$. They are constructed in such a way that the norm of $a_{i}$ is attained at each point of $A_{i}$, so that $a_{i}$ lies on an edge of $i / K . B_{c_{0}}$ (because $\left|A_{i}\right| \geq 2$ ). Therefore, the norm of $c_{0}$ is rough at the point $a_{i}$. Hence it is possible to select a direction $h_{i+1}$ very close to the kernel of $f^{\prime}\left(a_{i}\right)$ (so that $f\left(a_{i}+h_{i+1}\right)-f\left(a_{i}\right)$ is very small) such that $\left\|a_{i}+h_{i+1}\right\|-\left\|a_{i}\right\|$ is large (actually $\left.\left\|a_{i}+h_{i+1}\right\|-\left\|a_{i}\right\|=\left\|h_{i+1}\right\|=1 / K\right)$. Moreover, in order to iterate the construction, the direction $h_{i+1}$ should be selected in such a way that $a_{i+1}=a_{i}+h_{i+1}$ lies on an edge of $(i+1) / K . B_{c_{0}}$, which explains the choice of the sequence $\left(N_{0}, \ldots, N_{K}\right)$. Summing up, we obtain that $f\left(a_{K}\right)-f\left(a_{0}\right)$ is small and $\left\|a_{K}\right\|-\left\|a_{0}\right\|=1$ is "very" large.

Theorem 1. Assume that $Y$ has finite cotype and that $X$ does not have finite cotype, and let $\Omega$ be a bounded open subset of $X$. Then, for any function $f \in$ $C_{u}^{1}(\bar{\Omega}, Y), f(\partial \Omega)$ is dense in $f(\bar{\Omega})$.

Proof. By the Maurey-Pisier theorem, $X$ contains $l_{\infty}^{n}$ 's uniformly. Thus, it should be clear that Theorem 1 follows easily from Lemma 1 . We give the details anyway.

Let $f \in C_{u}^{1}(\bar{\Omega}, Y)$, and denote by $\omega$ be the modulus of uniform continuity of $f^{\prime}$ on $\bar{\Omega}$. Choose $C>0$ such that $\forall x \in \Omega x+C B_{X} \supseteq \Omega$ and let $M=2 C \sup \left\{\left\|f^{\prime}(x)\right\| ; x \in\right.$ $\bar{\Omega}\}$. Finally, let $\varepsilon>0$, choose a positive integer $K$ such that $\omega(1 / K)<\varepsilon / 2$, and let $N$ be an integer satisfying the conclusion of Lemma 1 for $\omega, M, K$ and $\varepsilon / 2$.

Since $X$ contains $l_{\infty}^{n}$ 's uniformly, we can choose a linear embedding $T: l_{\infty}^{N} \rightarrow X$ such that $\|T\| \leq 2 C$ and $\left\|T^{-1}\right\| \leq 1 / C$.

Now, let $x_{0} \in \Omega$ and put $V=\left\{u \in l_{\infty}^{N} ; x_{0}+T u \in \Omega\right\}$.

The set $V$ is an open neighbourhood of 0 in $l_{\infty}^{N}$, and it is contained in $B_{l_{\infty}^{N}}$ because $\left\|T^{-1}\right\| \leq 1 / C$. Moreover, the function $\tilde{f}$ defined by $\tilde{f}(u)=f\left(x_{0}+T u\right)$ is in $C^{1, \omega, M}(\bar{V}, Y)$. Therefore, by the choice of $N$, there exists a point $a \in \partial V$ such 
that $\|\tilde{f}(a)-\tilde{f}(0)\|<\varepsilon$. Then $x=x_{0}+T a$ belongs to $\partial \Omega$, and $\left\|f(x)-f\left(x_{0}\right)\right\|<\varepsilon$. This concludes the proof.

Definition 2. Let $N$ be a positive integer, and let $\mathcal{S}_{N}$ be the permutation group of $\{0 ; \ldots ; N-1\}$. A function $f: c_{0} \rightarrow Y$ is said to be $\mathcal{S}_{N}$-invariant if for each $\sigma \in \mathcal{S}_{N}$ and all $x \in c_{0}$, one has $f\left(x_{\sigma}\right)=f(x)$, where $x_{\sigma}=\left(x_{\sigma(0)}, \ldots, x_{\sigma(N-1)}, x_{N}, x_{N+1}, \ldots\right)$.

Lemma 2. Assume that $Y$ has finite cotype. Let $\omega$ be a modulus of continuity, and let $M>0$. Finally, let $K$ be a positive integer and let $\varepsilon>0$.

a) Given a finite set $F \subseteq B_{c_{00}}$, there exists a positive integer $N$ satisfying the following property: for every $f \in C^{1, \omega, M}\left(B_{c_{0}}, Y\right)$, one can find a normalized $K$-pyramidal vector a whose support is contained in $[0 ; N[$ and disjoint from $\bigcup_{x \in F} \operatorname{supp} x$, and such that

$$
\forall x \in F \quad\|f(x+a)-f(x)\|<\varepsilon+\omega(1 / K) .
$$

b) Let $F=\left\{0 ; e_{0}\right\}$. If $K$ is large enough and if $N$ is chosen as in $\left.\mathbf{a}\right)$, then $\| f\left(e_{0}\right)-$ $f(0) \|<3 \varepsilon$ for each $\mathcal{S}_{N}$-invariant $f \in C^{1, \omega, M}\left(B_{c_{0}}, Y\right)$.

Proof. a) Let $F=\left\{x_{1} ; \ldots ; x_{m}\right\}$ be a finite subset of $B_{c_{00}}$, and choose an integer $L$ such that $\bigcup_{i=1}^{m} \operatorname{supp} x_{i} \subseteq[0 ; L]$. Let also $\tilde{Y}=l_{\infty}^{m}(Y)$. Finally, let $T$ be the "rightshift" operator on $c_{0}$, defined by $T\left(\sum \alpha_{i} e_{i}\right)=\sum \alpha_{i} e_{i+1}$.

Then, for any $f \in C^{1, \omega, M}\left(B_{c_{0}}, Y\right)$, the function $\tilde{f}$ defined by

$$
\tilde{f}(u)=\left(f\left(x_{1}+T^{L+1} u\right), \ldots, f\left(x_{m}+T^{L+1} u\right)\right)
$$

is in $C^{1, \omega, M}\left(B_{c_{0}}, \tilde{Y}\right)$. Therefore, part a) follows from Lemma 1 applied to $\tilde{Y}$.

b) Choose an integer $N$ satisfying the conclusion of a) for $F=\left\{0 ; e_{0}\right\}$, and let $f \in C^{1, \omega, M}\left(B_{c_{0}}, Y\right)$ be $\mathcal{S}_{N}$-invariant.

By a), one can find a normalized $K$-pyramidal vector $a=\frac{1}{K} \sum_{i=1}^{K} \mathbf{1}_{A_{i}}$ supported by $] 0 ; N[$ such that

$$
\|f(a)-f(0)\|,\left\|f\left(e_{0}+a\right)-f\left(e_{0}\right)\right\|<\varepsilon+\omega(1 / K) .
$$

Let $i_{0}=\min A_{K}$, and put $h=\frac{1}{K} \sum_{i=1}^{K} e_{l_{i}}$, where $l_{i}=\min A_{i}-1(1 \leq i \leq K)$. Then $\|h\|=1 / K$ and $a, a+h \in B_{c_{0}}$; hence $\|f(a+h)-f(a)\| \leq M / K$. But $a+h=\left(a+e_{0}\right)_{\sigma}$, where $\sigma \in \mathcal{S}_{N}$ is the cycle $\left(0, \ldots, i_{0}\right)$. Therefore (by $\mathcal{S}_{N}-$ invariance) $f(a+h)=f\left(a+e_{0}\right)$, whence $\left\|f\left(a+e_{0}\right)-f(a)\right\| \leq M / K$. By the choice of $a$, it follows that

$$
\begin{aligned}
\left\|f\left(e_{0}\right)-f(0)\right\| & \leq\left\|f\left(e_{0}\right)-f\left(e_{0}+a\right)\right\|+\left\|f\left(e_{0}+a\right)-f(a)\right\|+\|f(a)-f(0)\| \\
& \leq M / K+2(\epsilon+\omega(1 / K)) .
\end{aligned}
$$

This proves $\mathbf{b})$.

Theorem 2. Assume that $Y$ has finite cotype.

a) If $f \in C_{u}^{1}\left(B_{c_{0}}, Y\right)$, then the sequence $\left(f\left(e_{i}\right)\right)$ converges to $f(0)$ in the Cesaro sense. 
b) More precisely, given a modulus of continuity $\omega$ and positive numbers $M$, $\varepsilon$, there exists a positive integer $N$ such that $\left\|\frac{1}{n} \sum_{i=0}^{n-1} f\left(e_{i}\right)-f(0)\right\|<\varepsilon$ for all $f \in C^{1, \omega, M}\left(B_{c_{0}}, Y\right)$ and all $n \geq N$.

Proof. Let $\omega$ be a modulus of continuity, and let $M>0$. If $f \in C^{1, \omega, M}\left(B_{c_{0}}, Y\right)$, then, for any positive integer $n$, the function $\tilde{f}$ defined by $\tilde{f}(x)=\frac{1}{n !} \sum_{\sigma \in \mathcal{S}_{n}} f\left(x_{\sigma}\right)$ still belongs to $C^{1, \omega, M}\left(B_{c_{0}}, Y\right)$, and it is also $\mathcal{S}_{n}$-invariant; moreover, $\tilde{f}(0)=f(0)$ and $\tilde{f}\left(e_{0}\right)=\frac{1}{n} \sum_{i=0}^{n-1} f\left(e_{i}\right)$. Thus, Theorem 2 follows from Lemma 2 .

Corollary. Assume that $Y$ has finite cotype, and let $f: c_{0} \rightarrow Y$ be a $C^{1}$-smooth function such that $f^{\prime}$ is uniformly continuous on bounded sets. Then any sequence $\left(x_{i}\right) \subseteq c_{0}$ weakly converging to some $x \in c_{0}$ has a subsequence $\left(x_{i}^{\prime}\right)$ such that $f\left(x_{i}^{\prime}\right) \rightarrow$ $f(x)$ in the Cesaro sense.

Remark. Clearly, the conclusion of Theorem $2 \mathbf{b})$ holds for any subsequence of $\left(e_{i}\right)$, with the same integer $N$.

It is very likely that Theorem 2 is far from being best possible. An "optimal" statement could be the following: if $Y$ does not contain $c_{o}$ and $f \in C_{u}^{1}\left(B_{c_{0}}, Y\right)$, then $f$ turns weak-Cauchy sequences from $B_{c_{0}}$ into norm convergent sequences in $Y$.

Notice that if $Y$ has an unconditional basis, then the above statement is indeed true, as shown in $\mathrm{H} 2$.

In the same spirit, given a pair of Banach spaces $(X, Y)$ and a function $f \in$ $C^{1}\left(B_{X}, Y\right)$, one may consider the following two properties:

(1) $f$ turns Cauchy sequences (from $B_{X}$ ) for the "weak" topology generated by $\mathcal{L}(X, Y)$ into (norm) convergent sequences.

(2) $f^{\prime}\left(B_{X}\right)$ is a relatively compact subset of $\mathcal{L}(X, Y)$.

It follows at once from the mean-value theorem that property (2) is stronger than (1). Moreover, it is observed in $[\mathrm{H} 2$ that when $Y=\mathbb{R},(\mathbf{1})$ are (2) are equivalent provided $X$ does not contain $l_{1}$ and $f^{\prime}$ is uniformly continuous on $B_{X}$. Finally, if $Y=\mathbb{R}$ and $X=c_{0}$, then both properties are true; this is the main result of [H1].

In the remainder of this note, we give a short proof of a slightly weaker form of this last result (Theorem 3 below).

For the sake of readability, we will impose "global" smoothness conditions on the functions we are dealing with. Accordingly, we shall say that a function $f: c_{0} \rightarrow Y$ is smooth if $f$ is $C^{1}$-smooth and $f^{\prime}$ is uniformly continuous on bounded sets.

Definition 3. Let $(G,+)$ be an abelian topological group, and let $B$ be a subset of $G$. We say that a function $f: G \rightarrow Y$ is strongly sequentially continuous in $B$ if for every sequence $\left(x_{n}\right) \subseteq B$ and every sequence $\left(h_{i}\right)$ converging to 0 in $G$, one has

$$
\lim _{i \rightarrow \infty}\left(\liminf _{n \rightarrow \infty}\left\|f\left(x_{n}+h_{i}\right)-f\left(x_{n}\right)\right\|\right)=0 .
$$

It is easily checked that the definition of strong sequential continuity can be reformulated as follows: a function $f: G \rightarrow Y$ is strongly sequentially continuous 
in a set $B$ if and only if, for each sequence $\left(h_{i}\right)$ converging to 0 in $G$, the sequence of functions $\left(f_{n}\right)$ defined by $f_{n}(x)=\inf \left\{\left\|f\left(x+h_{i}\right)-f(x)\right\| ; i \leq n\right\}$ converges to 0 uniformly on $B$.

This definition may look a bit artificial. It is "justified" by the following lemma.

Lemma 3. Let $G$ be an abelian topological group and let $f: G \rightarrow Y$. Assume that $f$ is strongly sequentially continuous in some set $B \subseteq G$. Then the following statements hold.

a) $f$ turns Cauchy sequences from $B$ into (norm) convergent sequences in $Y$.

b) If, in addition, every sequence from $B$ admits a Cauchy subsequence, then $f_{\mid B}$ is uniformly sequentially continuous on $B$.

Proof. a) By contradiction, assume that there exists a Cauchy sequence $\left(x_{i}\right) \subseteq B$ such that $\left(f\left(x_{i}\right)\right)$ is not convergent. Then we can find a positive number $\varepsilon$ and two subsequences $\left(y_{n}\right),\left(z_{m}\right)$ of $\left(x_{i}\right)$ such that $\forall n, m \geq 0\left\|f\left(y_{n}\right)-f\left(z_{m}\right)\right\| \geq \varepsilon$; this is obvious if the set $\left\{f\left(x_{i}\right) ; i \geq 0\right\}$ is not relatively compact in $Y$ (because in this case, $\left(f\left(x_{i}\right)\right)$ admits an $\varepsilon$-separated subsequence), and obvious as well if it is, because in that case, $\left(f\left(x_{i}\right)\right)$ has at least two cluster points.

Now, for all $i, n, m \geq 0$, one has

$$
\left\|f\left(y_{n}\right)-f\left[y_{n}+\left(z_{m}-x_{i}\right)\right]\right\|+\left\|f\left[z_{m}+\left(y_{n}-x_{i}\right)\right]-f\left(z_{m}\right)\right\| \geq \varepsilon
$$

Thus, by Ramsey's theorem for triples of integers, we may assume that either $\forall i, n, m \in \mathbb{N}, m<i<n,\left\|f\left(y_{n}\right)-f\left[y_{n}+\left(z_{m}-x_{i}\right)\right]\right\| \geq \varepsilon / 2$, or $\forall i, n, m \in \mathbb{N}$, $m<i<n,\left\|f\left[z_{m}+\left(y_{n}-x_{i}\right)\right]-f\left(z_{m}\right)\right\| \geq \varepsilon / 2$. In the first case, we get in particular $\liminf _{n \rightarrow \infty}\left\|f\left(y_{n}\right)-f\left[y_{n}+\left(z_{i-1}-x_{i}\right)\right]\right\| \geq \varepsilon / 2$ for all $i \geq 1$, which is impossible because $\left(z_{i-1}-x_{i}\right) \rightarrow 0$ as $i \rightarrow \infty$ and $f$ is strongly sequentially continuous in $B$. In the second case, we get $\left\|f\left[z_{0}+\left(y_{i+1}-x_{i}\right)\right]-f\left(z_{0}\right)\right\| \geq \varepsilon / 2$ for all $i \geq 1$, which is again impossible because $f$ is sequentially continuous at $z_{0}$. This proves a).

Part b) is a straightforward consequence of $\mathbf{a}$ ).

The following remarks will not be used in the proof of Theorem 3 .

Remarks. 1. Clearly, strong sequential continuity in $B \subseteq G$ implies sequential continuity at each point of $B$. Moreover, it is not difficult to check that if $f: \mathbb{R} \rightarrow \mathbb{R}$ is strongly sequentially continuous in $\mathbb{R}$, then $\liminf _{|x| \rightarrow \infty}|f(x) / x|<+\infty$. Thus, strong sequential continuity in $\mathbb{R}$ is a stronger property than usual continuity.

2. It is plain that any uniformly sequentially continuous function $f: G \rightarrow Y$ is strongly sequentially continuous in $G$. On the other hand, Lemma 3 implies that if $f: \mathbb{R} \rightarrow Y$ is strongly sequentially continuous in some bounded set $B \subseteq \mathbb{R}$, then $f_{\mid B}$ is uniformly continuous on $B$. More generally, if $G=(X, w)$, where $X$ is a Banach space not containing $l_{1}$, then strong sequential continuity in bounded sets is equivalent to sequential uniform continuity on bounded sets, by Rosenthal's $l_{1}$-theorem.

3. We are unable to determine whether strong sequential continuity in $\mathbb{R}$ is equivalent to uniform continuity.

4. The following example shows that even in very simple groups, strong sequential continuity does not imply uniform continuity.

Let $\mathbb{D}$ be the group of dyadic real numbers $\left(\mathbb{D}=\left\{k / 2^{p} ; k \in \mathbb{Z}, p \in \mathbb{N}\right\}\right)$, and let $f: \mathbb{D} \rightarrow \mathbb{R}$ be the even function defined on $\mathbb{D} \cap[n, n+1](n \in \mathbb{N})$ by 
$f(x)=\sin (\pi x) \sin \left(2^{n} \pi x\right)$. It is easy to check that $f$ is not uniformly continuous on $\mathbb{D}$. Yet, we claim that $f$ is strongly sequentially continuous in $\mathbb{D}$.

To show this, let us fix $\varepsilon>0$ and a sequence $\left(h_{i}\right) \subseteq \mathbb{D}$ converging to 0 . It is enough to prove that for large enough $n, \sup _{x \in \mathbb{D}_{+}} \inf _{i \leq n}\left|f\left(x+h_{i}\right)-f(x)\right| \leq 2 \pi \varepsilon$ (where $\mathbb{D}_{+}=\mathbb{D} \cap[0 ;+\infty[)$.

First, we choose $n_{0}$ such that $\left|h_{n_{0}}\right|<\varepsilon / 2$, and we write $h_{n_{0}}=k_{0} / 2^{p_{0}}\left(p_{0} \in \mathbb{N}\right.$, $\left.k_{0} \in \mathbb{Z}\right)$.

Since $f$ is uniformly continuous around $\left[0 ; p_{0}+1\right]$, there exists an integer $n_{1}$ such that

$$
\forall x \in \mathbb{D} \cap\left[0 ; p_{0}+1\right]\left|f\left(x+h_{n_{1}}\right)-f(x)\right| \leq \varepsilon .
$$

Next, if $x \in[n-\varepsilon / 2, n+\varepsilon / 2]$ for some $n \in \mathbb{N}$, then $x+h_{n_{0}} \in[n-\varepsilon, n+\varepsilon]$; so

$$
\left|f\left(x+h_{n_{0}}\right)-f(x)\right| \leq\left|f\left(x+h_{n_{0}}\right)\right|+|f(x)| \leq 2 \pi \varepsilon
$$

for all $x \in \mathbb{D}_{+} \cap\left(\bigcup_{n \in \mathbb{N}}[n-\varepsilon / 2, n+\varepsilon / 2]\right)$.

Finally, if $x \in \mathbb{D}_{+} \backslash\left(\left[0 ; p_{0}+1\right] \cup \bigcup_{n \in \mathbb{N}}[n-\varepsilon / 2, n+\varepsilon / 2]\right)$, then there exists $n \geq p_{0}+1$ such that both $x$ and $x+h_{n_{0}}$ lie in $[n, n+1]$. Since $n \geq p_{0}+1$, the function $t \mapsto \sin \left(2^{n} \pi t\right)$ is $h_{n_{0}}$ periodic; hence

$$
f\left(x+h_{n_{0}}\right)-f(x)=\sin \left(2^{n} \pi x\right)\left[\sin \pi\left(x+h_{n_{0}}\right)-\sin (\pi x)\right] .
$$

Thus

$$
\left|f\left(x+h_{n_{0}}\right)-f(x)\right| \leq\left|\sin \pi\left(x+h_{n_{0}}\right)-\sin (\pi x)\right| \leq \pi \varepsilon / 2
$$

for all $x \in \mathbb{D}_{+} \backslash\left(\left[0 ; p_{0}+1\right] \cup \bigcup_{n \in \mathbb{N}}[n-\varepsilon / 2, n+\varepsilon / 2]\right)$.

Therefore, if $n \geq \operatorname{Max}\left(n_{0}, n_{1}\right)$, we have, for all $x \in \mathbb{D}_{+}$,

$$
\inf _{i \leq n}\left|f\left(x+h_{i}\right)-f(x)\right| \leq \operatorname{Max}(\varepsilon, \pi \varepsilon / 2,2 \pi \varepsilon) \leq 2 \pi \varepsilon .
$$

After this detour, we can now state and prove the following result:

Theorem 3 (Hájek). Let $f: c_{0} \rightarrow \mathbb{R}$ be a smooth function. Then $f$ is uniformly continuous on bounded sets when $c_{0}$ is equipped with its weak topology.

Proof. The weak topology is metrizable on any bounded subset of $c_{0}$, and each bounded sequence in $c_{0}$ admits a weak-Cauchy subsequence; hence, by Lemma 3 , we may content ourselves with proving that $f$ is strongly sequentially continuous in every bounded subset of $G=\left(c_{0}, w\right)$. Therefore, we have to show that if $\left(h_{i}\right) \subseteq c_{0}$ is weakly null, then $\inf \left\{\left\|f\left(x+h_{i}\right)-f(x)\right\| ; i \leq n\right\} \rightarrow 0$ uniformly on bounded sets.

Let us fix a weakly null sequence $\left(h_{i}\right)$ and a bounded set $B \subseteq c_{0}$.

By extracting a subsequence if necessary, we may assume that there exists a bounded linear operator $T: c_{0} \rightarrow c_{0}$ such that $T\left(e_{i}\right)=h_{i}$ for all $i$.

Let $\omega_{0}$ be the modulus of uniform continuity of $f^{\prime}$ on $B+T B_{c_{0}}$, and let $M_{0}=$ $\sup \left\{\left\|f^{\prime}(w)\right\| ; w \in B+T B_{c_{0}}\right\}$. Then, for every $x \in B$, the function $f_{x}$ defined by $f_{x}(u)=f(x+T u)$ is in $C^{1, \omega, M}\left(B_{c_{0}}, \mathbb{R}\right)$ where $\omega=\|T\| \cdot \omega_{0}$ and $M=\|T\| \cdot M$; 
hence, by Theorem 2 (and the remark following it),

$$
\lim _{n \rightarrow \infty}\left(\sup \left\{\left|\frac{1}{n} \sum_{i \in F}\left[f\left(x+h_{i}\right)-f(x)\right]\right| ; F \subseteq \mathbb{N},|F|=n\right\}\right)=0 \text { uniformly on } B .
$$

Since $f$ is real-valued, this concludes the proof.

Corollary (Hájek). If $f: c_{0} \rightarrow \mathbb{R}$ is smooth, then $f^{\prime}$ is a compact map, which means that for every bounded set $B \subseteq c_{0}, f^{\prime}(B)$ is relatively compact in $l_{1}$.

Proof. We could apply the result mentioned above about the equivalence of properties (1) and (2), but we give a direct proof for completeness.

Let $f: c_{0} \rightarrow \mathbb{R}$ be a smooth function, and assume that for some bounded $B \subseteq c_{0}$, $f^{\prime}(B)$ is not relatively compact in $l_{1}$. Then one can find a positive number $\varepsilon$, a sequence $\left(x_{i}\right) \subseteq B$ and a sequence $\left(h_{i}\right) \subseteq B_{c_{0}}$, such that the $h_{i}$ 's are disjointly supported and $\left|f^{\prime}\left(x_{i}\right) . h_{i}\right| \geq \varepsilon$ for all $i$.

Let $\omega$ be the modulus of (uniform) continuity of $f^{\prime}$ on $B+B_{c_{0}}$, and fix $\left.\left.\alpha \in\right] 0 ; 1\right]$. Then, for each $i \geq 0$, one can write

$$
\begin{aligned}
\left|f\left(x_{i}+\alpha h_{i}\right)-f\left(x_{i}\right)\right| & \geq\left|f^{\prime}\left(x_{i}\right) .\left(\alpha h_{i}\right)\right|-\left\|\alpha h_{i}\right\| \omega\left(\left\|\alpha h_{i}\right\|\right) \\
& \geq \alpha(\varepsilon-\omega(\alpha)) .
\end{aligned}
$$

Since $\left(h_{i}\right)$ is weakly null, this contradicts Theorem 3 if $\alpha$ is small enough.

To conclude this note, let us mention still another innocent question.

Theorem 3 implies that any smooth function $f: c_{0} \rightarrow \mathbb{R}$ can be (uniquely) extended to a function $\tilde{f}: l_{\infty} \rightarrow \mathbb{R}$ which is $w^{*}$-continuous on bounded sets. It would be interesting to know if such an extension inherits any smoothness property from $f$.

\section{REFERENCES}

[B] S. M. Bates, On smooth, nonlinear surjections of Banach spaces, Israel J. Math. 100 (1997), 209-220. MR 98i:58016

[H1] P. Hájek, Smooth functions on $c_{0}$, Israel J. Math. 104 (1998), 17-27. MR 99d:46063

[H2] P. Hájek, Smooth functions on $C(K)$, Israel J. Math. 107 (1998), 237-252. CMP 99:05

Université Bordeaux 1, 351, Cours de la libération, 33405 Talence Cedex, France

E-mail address: deville@math.u-bordeaux.fr

E-mail address: matheron@math.u-bordeaux.fr 